\title{
Editorial
}

\section{Physical and Recreational Activity for Active Ageing}

\section{Haque M Md*}

Organizing Secretary, Bangladesh Physical Therapy

Association, Dhaka, Bangladesh

*Corresponding author: Md Monoarul Haque, Organizing Secretary, Bangladesh Physical Therapy Association, Dhaka, Bangladesh

Received: March 16, 2018; Accepted: April 27, 2018;

Published: May 04, 2018

\section{Editorial}

Geriatric people are increasing in Bangladesh. As a result, musculoskeletal problems are also increasing in full swing. Technology dependent or sedentary life style is much more responsible behind this vivid scenario. Now voice is rising for active ageing. In order to achieve fit ageing, no alternative exists without physical exercise. We know physical exercise keeps one healthy; recreational activities bring mental happiness. Older group needs both of these activities to keep them free from disease. But we often show negative attitude to physical exercise as well as recreational activity particularly in later part of life. WHO, 2004 [1] stated that pattern of diseases among the senior citizen in South-East Asia has led to change due to increase life span, changes in life style and environment. Inappropriate eating and lack of physical exercise are rated as second and third leading health risk behavior in Bangladesh WHO, 2009 [2]. We know geriatric population is a vulnerable segment of a society because they can break with a slightest stimulus though they occupy wide and greater portion of population pyramid. Due to improvement of health facility life span of Bangladeshi people has increased. But this high life span brings disease burden also such as musculoskeletal problem along with risk of other co-morbidities like high blood pressure, diabetes and cerebrovascular accident are increasing rapidly. Quality adjusted life year is going down but disability adjusted life year is expanding. But solution is in own hand i.e. physical activity. WHO, 2010 [3] recommends adults aged 65 years and older to engage in at least 150 minutes aerobic PA at moderate intensity or 75 minutes aerobic PA at vigorous intensity per week, or an equivalent combination of aerobic PA at moderate and vigorous intensity. To obtain additional health benefits, aerobic PA has to be increased up to 300 minutes at moderate intensity or 150 minutes at vigorous intensity per week. In addition to aerobic PA, muscle strengthening of major muscle groups should be performed on 2 or more days per week. Older adults with poor mobility should perform exercises specific for improved balance on 3 or more days per week. Havighurst [4] found the relationship between leisure activities and wellbeing in later life. He showed that maintained activity was positively related to the wellbeing of senior citizen. DiPietro [5] proved that active life style lowers risk of noncommunicable diseases i.e. heart disease, stroke, type 2 diabetes, cancers, depression and dementia.

\section{References}

1. WHO. Health of the Elderly in South-East Asia. 2004.

2. WHO. Global Health Risks: mortality and burden of disease attributable to selected major risks. 2009.

3. World Health Organization. Global Recommendations on Physical Activity for Health. Geneva, Switzerland: WHO Press. 2010.

4. Havighurst, R. The nature and values of meaningful free-time activity, in R Kleemeier. Aging and Leisure Edn. New York: Oxford University Press. 1961 309-344.

5. DiPietro, L. Physical Activity in Aging: Changes in Patterns and Their Relationship to Health and Function. J Gerontol A Biol Sci Med Sci. 2001; 56:13-22. 